\title{
ADAPTIVE APPROACH TO RETRIEVE IMAGE AFFECTED BY IMPULSE NOISE
}

\author{
Gururaj P.Surampalli ${ }^{1}$, Bhimrao Patil $^{2}$, Giriraj Patil ${ }^{3}$ \\ ${ }^{1}$ Asst. Professor, Dept. of Computer Science and Engineering, GNDEC, Bidar 585 401, \\ ${ }^{2}$ Asst. Professor, Dept. of Computer Science and Engineering, GNDEC, Bidar 585401 \\ ${ }^{3}$ Asst. Professor, Dept. of Computer Science and Engineering, GNDEC, Bidar 585401
}

\begin{abstract}
Impulsive noise is a kind of noise in sim literature it is also called salt-and-pepper noise or spike noise is most oftenly occuring image detoriation. It is a type of noise which has black pixel in white region and white pixel in black region, this type of noise can be caused by almost lost value of pixels, inefficient analog-to-digital converter, change in bit value during transmission eavesdrops, fault and failure in communication etc. The traditional filtering techniques such as mean median and average are available to remove the noise from an image one of the most oftenly used is Gaussian filtering technique. But these filtering techniques are not generic, where as such techniques are useful in preparing adaptive algorithm for the general purpose filters.
\end{abstract}

\section{INTRODUCTION}

Digital image processing is a tree with many advanced applications like robotics, medical imaging, computer vision, feature detection and remote sensing as fruits.

It has many roots such as image acquisition, image restoration, color image processing, image morphology, image segmentation etc.

In this paper, there are topics associated with the image restoration were discussed such as cause for impulse noises in image, filtering techniques used to restore the images.

Image is a proper organization of the pixels. Each pixel can be represented as two dimensional function. Image processing includes changing the image nature or characteristic for

1) Improve interpretation of image so as to it can be judged by human

2) Apply it more suitable for independent machine acceptance Image acquisition is the first process in image processing; it includes preprocessing steps such as scaling where image resize is done.

Image enhancement is among the simple and important digital image processing fields or areas, its purpose is to bring important information which is changed and emphasizing on certain content which are crucial. Image restoration concerns the removal or reduction of pixel value which have taken place during the acquisition of image. Such deterioration of the image may include noise, which are distraction in the pixel values, or optical effect such as out of focus blurring, or blurring due to camera motion. We shall see that some restoration techniques can be performed very successfully using nearby neighborhood operations, while some method require the use of frequency domain processes.

One of the important field of image processing is restoration of image, but in this paper the emphasis will be on the techniques for dealing with restoration, and degradations types, or the properties of electronic equipment which give rise to image degradation.

In addition to this there are other steps in digital image processing such as acquisition, color processing, wavelet, compression etc are some most often used steps in image processing. In image restoration process the output generally are images, as in object recognition the outputs are the attributes of images.

\section{IMPULSE NOISE}

Image processing is one of the ordinary component in modern science and it includes many important applications. With the expanding use of different size and orientation images in daily-life applications is challenging, in such scenario image quality researchers are rapidly improving their algorithm. But some errors may have larger impact on the image pixel, during image acquisition, transmission and storage etc are some of them.

Impulsive Noise (IN) takes place due to the transmission medium non linearity, transmission system errors, sensor motion at the time of image exposure, fault in memory-sensor units, imperfections of electro-optic cross, electromagnetic interferences, storage medium size and image processing methods. An impulse noise is also called salt and pepper noise and some other names as shot noise, binary noise. These noise 
caused by sharp, rapid disturbances in the image signal which alters pixel value. It appears as it randomly scattered white or black (or both) pixels over space of the image.

The PDF(probability density function) related to impulse noise given by following function

$$
p(z)= \begin{cases}P_{a} & \text { for } z=a \\ P_{b} & \text { for } z=b \\ 0 & \text { Otherwise }\end{cases}
$$

If $\mathrm{b}>\mathrm{a}$, gray-level $\mathrm{b}$, which appear on image as a light dot.

Conversely, level a which appear as dark dot. If either Pa or $\mathrm{Pb}$ is zero, the impulse noise such situation is called unipolar. If neither probability is zero and specially if they are approximately equal, impulse noise values appears as salt-andpepper granules randomly spread over the image. For such a reason, bipolar impulse noise also known as salt-and-pepper. Shot and spike noise also used to refer this type of noise. In our discussion we will use the terms impulse or salt-andpepper noise interchangeably.

Noise impulses can be one of the negative or positive. Scaling is important part of the image digitizing process. Because image detoriation usually is part of the image digitizing process. Because impulse corruption is high compared with the strength of the image signal, impulse noise usually is digitized as extreme (pure black or white) values in an image. Thus, the assumption usually is that a and b are "saturated" values, means that they are equal to the minimum and maximum are allowed pixel values in the digitized image. As a result, negative impulses looks as black (pepper) dots in an image. For the similar reason, positive impulses looks as white (salt) noise. For an 8-bit test image it means such as a $=0$ (black) and $b=255$ (white).

Graphically it is shown below.
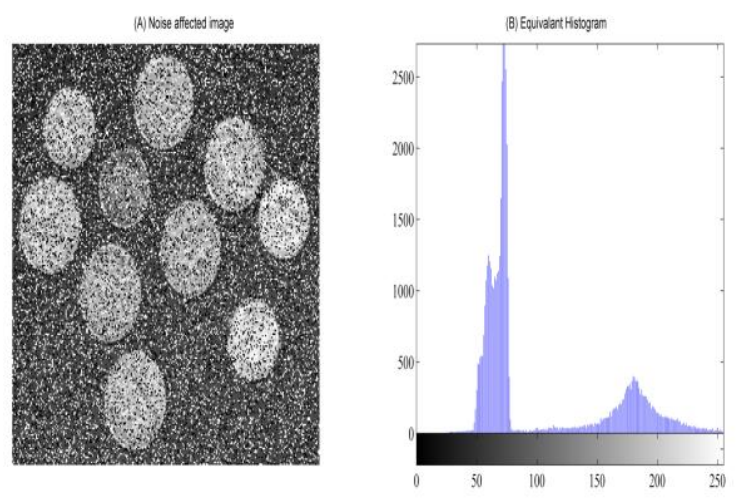

Adaptive filters are a class of filters which change their characteristics according to the values of the grey scales under the mask in the matrix, they may act more like median filters, or more like average filters. Depending on their position within the image matrix. Such a filter can be used to clean Gaussian noise by using local statistical properties of the values under the matrix mask.

Salt-and-pepper impulse noise is one the most often and commonly occurring noise type during video communication. So far there are other methods can reasonably restore images corrupted or affected by salt-and-pepper noise whose level is up to $70 \%$
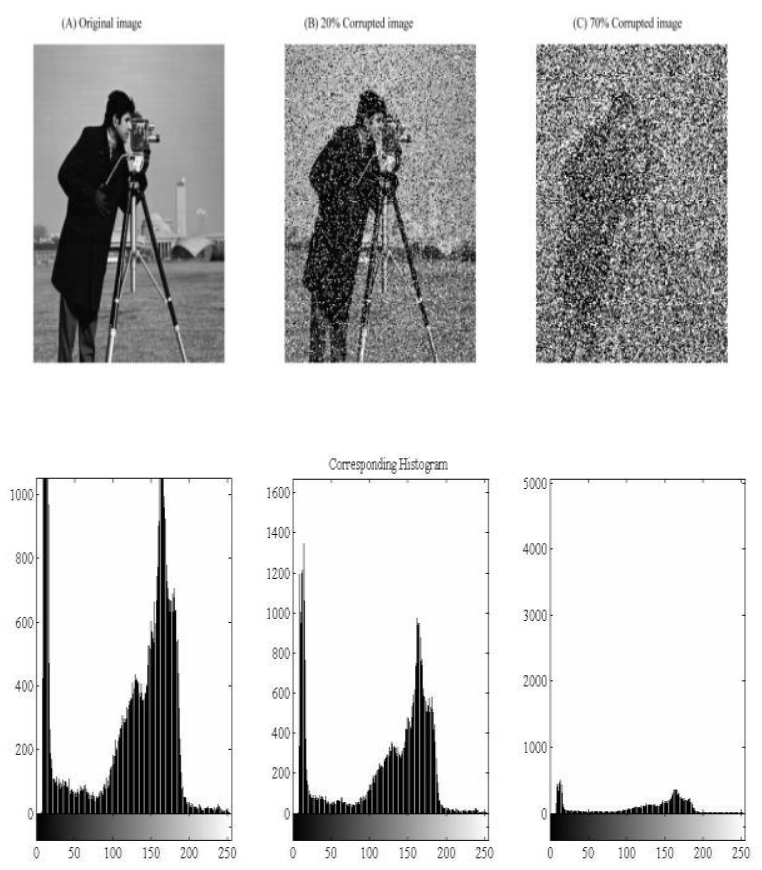

\section{FILTERS}

\subsection{Mean Filtering}

To recover the image which is corrupted with noise there many available mean filtering techniques and they are application oriented method. Some filtering techniques have better or good effects than the others according to different noise category and type. Mean filtering techniques are described as below

\subsubsection{Arithmetic Mean Filtering (AMF) Technique:}

This is one of the the simplest of the mean filtering techniques. Let Sxy represent the set of coordinates in a rectangular space that is image window of size $\mathrm{m} \times \mathrm{n}$ centered at given point $\quad(\mathrm{x}, \mathrm{y})$.

The AMF technique calculates the average value of the corrupted image $\mathrm{g}(\mathrm{x}, \mathrm{y})$ in the area defined by Sxy. The value 
of restored image $\mathrm{f}$ at any point $(\mathrm{x}, \mathrm{y})$ is Arithmetic Mean computed using the pixels values in the image that is in the region defined by Sxy. It can be expressed as AMF by the equation [4]

$$
f(x, y)=\frac{1}{m n} \sum_{(s, t) \in S_{x y}} g(s, t)
$$

\subsubsection{Geometric Mean Filtering (GMF) Technique:}

For GMF technique all restored pixel is given by the product of the pixel values in the sub image window under area, raised to the power $(1 / \mathrm{mn})$. A Geometric Mean Filter achieves output as smooth image comparable to the Arithmetic Mean Filter but it may lose less image quality during the process. GMF can be expressed by the equation given below[4]

$$
f(x, y)=\left[\prod_{(s, t) \in S_{x y}} g(s, t)\right]^{\frac{1}{m n}}
$$

\subsubsection{Harmonic Mean Filtering (HMF) Technique:}

The Harmonic Mean Filter works as efficient method for Salt noise and fails for Pepper noise. It does well with some other types of noise and most non linear noise like Gaussian noise. The HMF operation is given by the equation below [4]

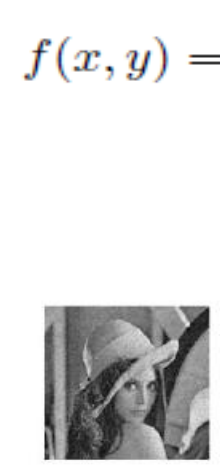

(a)

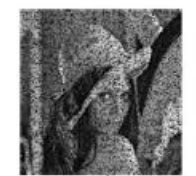

(b)

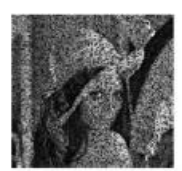

(c)

Fig. 3. Arithmetic mean filtering(AMF)(a), Geometric mean filtering(GMF) (b), Harmonic mean filtering (HMF) (c) [4]

\subsection{Median Filtering}

Median Filter is an image filter more effective or efficient in situations in which white spots and black spots appear on the test image. For this technique the center value of the mn window is considered to replace the black pixel and white pixels.

When white spots, black spots appear on the test image, it becomes very difficult to guess which pixel is the affected one. Replacing those disturbed or changed pixels with AMF, GMF and HMF are inefficient because those pixels are replaced by a value, it is not appropriate to the original pixel. We have observed over the distorted images that Median Filter has good influence than that of AMF, GMF and HMF, where $\mathrm{AMF}$ is the one of the best among all the mean filtering techniques and HMF is one of worst performance[4].

Median filtering seems almost tailor-made for removal of salt and pepper noise. Remember that the median of a set is the middle value when they are sorted. If there are an even number of values, the median is the mean of the middle two. A median filter is an example of a non-linear spatial filter. The operation of obtaining the median means that very high or very small values noise pixel values will end up at the top or bottom of the sequenced list. Thus the median will in general replace a noise value of pixel with one nearer to its surroundings.

In MATLAB, median filtering is implemented by the medfilt2 function:

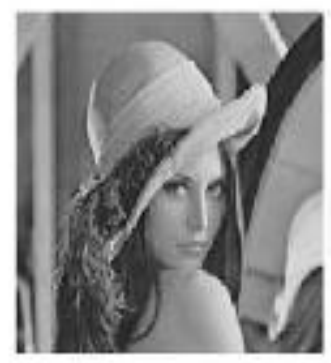

\section{Fig. 4. Filtered image with median filters [4]}

\subsection{Adaptive Filtering}

Adaptive filters are a class of filters which change their characteristics according to the values of the grey scales under the mask; they may act more like median filters, or more like average filters, depending on their position within the image. Such a filter can be used to clean Gaussian noise by using local statistical properties of the values under the mask.

One such filter is the minimum mean-square error filter; this is a non-linear spatial filter; and as with all spatial filters, is 
implemented by applying a function to the grey values under the mask. Since we are dealing with additive noise, our noisy image M' can be written as

$$
\mathrm{M}^{\prime}=\mathrm{M}+\mathrm{N}
$$

Here $\mathrm{M}$ is the original correct given test image and $\mathrm{N}$ is the added noise; which we assume to be normally distributed with mean 0 .

However, within our mask, the mean may not be zero; suppose the mean is $\mathrm{mf}$, and the variance in the mask is of 2 Suppose also that the variance of the noise over the entire image is known to be $\sigma \mathrm{g} 2$. Then the output value can be calculated as

$$
m_{f}+\frac{\sigma_{f}^{2}}{\sigma_{f}^{2}+\sigma_{g}^{2}}\left(g-m_{f}\right)
$$

Where $\mathrm{g}$ is the current value of the pixel in the noise affected image. Note if the local variance of 2 is high, then the fraction will be close to 1 , and the output close to the original image value $\mathrm{g}$ This is appropriate, as high variance implies high detail such as edges, which should be preserved. Conversely, if the local variance is low, such as in a background area of the image, the fraction is close to zero, and the value returned is close to the mean value[4][5].

\subsubsection{Adaptive Median Filtering:}

Conventional known median filtering approaches apply the median operation to each pixel unconditionally, that is, without considering whether it is not corrupted or corrupted. As a result, even the uncorrupted pixels are filtered, and this can cause image quality degradation. An intuitive solution to make this problem is to implement an impulse noise detection mechanism prior to applying filtering; hence, only those pixels identified as corrupted pixel which would undergo the filtering process, and those pixel identified as uncorrupted would remain at same value in image space. By using such noise detection mechanism or intelligence into the median filtering technique. The switching median filters had significant performance improvement in output image.

The most popular approaches for dealing with such noise have been based on median filtering and/or on the best class of order statistic filters that have emerged from the study of median filters. Recently, modification in the median filtering scheme have been shown predictable amount of good and better result, under various specific signal-noise models, to deliver improved performance relative to other corresponding conventional methods. For example it may include the minimum maximum exclusive mean filter (MMEM), Florencios, Signal dependent rank-order mean (SDROM) filter and conditional median filtering (CMF). These are the filters which have all rendered excellent performance, but at the price or cost of significant increase in computational complexity. The main problem is that characterizes all approaches of this type is that they involve some more computation to determine one or more of the local order statistics.

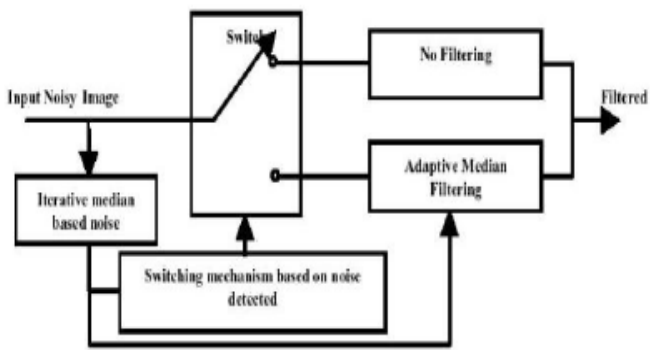

Fig. 5. Block diagram of adaptive median Filter [6]

\section{CONCLUSIONS}

In this proposed paper the two advanced filtering techniques were discussed namely linear filters which include arithmetic mean filter, geometric mean filter, harmonic mean filters etc, these have output images slightly blurred. In another technique namely non-linear filters such as median filter which has smooth output. The advanced adaptive technique also discussed which work better than the linear and nonlinear filter on impulse noise which is known as adaptive median filtering technique. These adaptive filters are very useful in many applications.

\section{REFERENCES}

[1]. K. Shahriar, R. Sakib, Jubayer, and R. Mizanur, "Salt and pepper noise detection and removal by tolerance based selective arithmetic mean filtering technique for image restoration," IJSNS International journal of computer science and network security. Vol8.No.6, pp. 271-278, 2009.

[2]. A. N. Rao, B. Rao, J. kiran, and L.S.S.Reddy, "A robust optimal morphological filter to remove impulse noise in images," International journal of Information Technology and knowledge Management Vol.2,No.2, pp. 237-239, Dec. 2010.

[3]. M. Alasdair, "An introduction to digital image processing with matlab(scm2511)," Victoria University of Technology, 2004.

[4]. P. Rajoo, "An improved switching median filter for uniformly distributed impulse noise removal," World academy of science, Engineering and technology 38, pp. 349-351, 2008.

[5]. S. Suryanarayana, D. B. L. Deekshatulu, D. K. L. Kishore, and Y. RakeshKumar, "Novel impulse detection technique for image denoising," Journal of theoretical and applied Information Technology, pp. 102-106, 2005-2009.

[6]. J. Mamta and M. Rajni, "An improved adaptive median filtering method for impulse noise detection," International journal of recent trends in engineering Vol.1, No.1, pp. 274-278, May 2009. 